\title{
On The Positive Expected Utility of Combination Wagers
}

\author{
Babatunde Buraimo, \\ David Peel \\ and \\ Rob Simmons
}

Authors' details: Corresponding author: Dr Babatunde Buraimo, Management School, University of Liverpool, Chatham Street, Liverpool, L69 7ZH, UK. Email:

b.buraimo@liverpool.ac.uk.

Professor David Peel, Management School, Lancaster University, Bailrigg, Lancaster, LA1 4YX, UK.

Professor Rob Simmons, Management School, Lancaster University, Bailrigg, Lancaster, LA1 4YX, UK. 


\title{
On The Positive Expected Utility of Combination Wagers
}

\begin{abstract}
We demonstrate that a utility maximizing individual with an everywhere concave utility function may optimally wager on two or more outcomes in an event even though the expected returns to a unit stake are negative on all outcomes except one.
\end{abstract}

\section{Keywords}

Expected utility, optimal wagering, combination wagers in an event.

\section{JEL Code}

D81, D84

We are grateful for very helpful comments from two referees and the Editor. 


\section{Introduction}

Bell (1998) sets out a surprising example where an expected utility (EU) maximizer, with an everywhere continuous and concave utility function, may undertake an actuarially unfair wager. The utility maximizer is near indifferent as to whether to accept or reject a binary wager which has a positive expected return but a given fixed stake. However, Bell demonstrates that the expected utility of a contingent strategy where the EU maximizer accepts the fixed stake wager if and only if he wins an independent actuarially unfair side bet may have higher expected utility than the original fixed stake wager. Consequently, the predecision side bet resolves the indifference towards the fixed stake wager. ${ }^{1}$

In this note we present another surprising example where an individual with EU preferences may optimally wager on an event outcome which has negative expected return. We demonstrate that a positive expected return to a unit bet on one outcome in an event can imply that it is utility maximizing to wager on a combination bet on two or more outcomes in the same event even when the expected returns to single unit bets on the other outcomes are negative. Though this possibility has been mentioned in previous literature, e.g. Phatarfod (2007), it has not been analyzed formally. We derive the optimal staking rules for an agent for wagering on two or three outcomes in an event assuming for simplicity that individuals have logarithmic utility functions. ${ }^{2}$

The rest of the letter is structured as follows. In section 2 we set out the optimal bets when wagering on two or three outcomes in an event when individuals have logarithmic utility functions. In section 3, we consider the properties of the combination wager relative to the single Kelly wager. Section 4 concludes. 


\section{Wagering on Two or Three Outcomes}

Assuming an agent's betting wealth is one unit, ${ }^{3}$ the expected utility, $U$, for an agent with a logarithmic utility function, betting on a single outcome with positive expected return is

$$
U=p \ln (1+j a)+(1-p) \ln (1-j)-\ln (1+i)
$$

where $j$ is the stake, $p$ is the probability of winning, $a$ is the odds quoted against the outcome occurring and $i$ is the safe interest rate. Differentiating (1) with respect to stake size $j$, we obtain the optimal stake as

$$
j=\frac{p a-(1-p)}{a}=\frac{\mu}{a}
$$

where $\mu$ is the expected return to a unit wager.

If the agent bets on two outcomes in sporting event e.g. a horse race, then expected logarithmic utility is given by

$$
U=p \ln (1+s a-v)+q \ln (l+v b-s)+(1-p-q) \ln (1-s-v)-\ln (1+i)
$$

where $s$ and $v$ are stakes, $p$ and $q$ are probabilities of the two outcomes, and $a$ and $b$ are odds. Differentiating (3) with respect to $s$ and $v$ we obtain the optimal stakes as

$$
\begin{aligned}
& S=\frac{b[p a-(1-p)]+q b-(1-q)}{a b-1}=\frac{\mu b+\lambda}{a b-1} \\
& v=\frac{a[q b-(1-q)]+p a-(1-p)}{a b-1}=\frac{\lambda a+\mu}{a b-1}
\end{aligned}
$$

where $\lambda$ is the expected return to a one unit bet on the second outcome. ${ }^{4}$ 
Consequently, the necessary conditions for the agent to bet on two outcomes are that

$$
a b-1>0, \mu b+\lambda>0, \text { and } \lambda a+\mu>0 .
$$

The second order condition for a maximum is given by

$$
a b-1-\mu(1+b)-\lambda(1+a)>0
$$

The second order condition ensures that total stake size, $s+v$, is less than total wealth of unity.

The interesting property revealed from (4) and (5) is that the EU maximizer may wager on two outcomes in an event even though the expected return for one of the outcomes is negative. This can occur since the expected return on the second outcome, $\lambda$ can be negative but both $\mu b+\lambda>0$ and $\lambda a+\mu>0$.

It is easy to show from (4) and (5) the perhaps surprising result that the stake, $s$, on the outcome for which expected return to a unit wager is positive could be less than the stake, $v$, on the outcome for which the expected return to a unit wager is negative. For example when, $\mu=0.25, a=10, \lambda=-0.01$ and $b=0.5$, the individual will wager 1.3 times as much on the favourite, which has a negative expected return to a unit wager, as on the outsider which has a positive expected return to a unit wager.

In principle, an expected utility maximizer could wager on $n-1$ outcomes in an event with $n>2$ outcomes. The formulae for wagering on three outcomes with stakes $s, v$ and $w$, odds 
$a, b$ and $c$ and expected returns of $\mu, \lambda$, and $\rho$ have closed form solutions for logarithmic utility and are given by

$$
\begin{aligned}
& s=\frac{\mu(b c-1)+\lambda(1+c)+\rho(1+b)}{a b c-a-b-c-2} \\
& v=\frac{\mu(1+c)+\lambda(c a-1)+\rho(1+b)}{a b c-a-b-c-2} \\
& w=\frac{\mu(1+b)+\lambda(1+a)+\rho(b a-1)}{a b c-a-b-c-2}
\end{aligned}
$$

with second order condition $s+v+w<1$. The formula for betting on more than three outcomes in a race do not have closed form solutions and would require solutions by a suitable algorithm.

\section{Some Properties of the Combination Wager Relative to the Kelly Wager}

We can derive a number of properties of the combination wager compared to the single Kelly wager. First, if it is optimal to wager on two outcomes in an event then the stake, $s$, on the outcome with the highest positive expected return to a unit wager will always be greater in the combination wager than in the optimal single Kelly wager, $j$. This follows since

$$
s-j=\frac{\mu b+\lambda}{a b-1}-\frac{\mu}{a}=\frac{a \lambda+\mu}{(a b-1) a}>0
$$

since $a \lambda+\mu>0$ and $a b-1>0$ in order to wager on two outcomes from (6).

Second, the expected return from the optimal combination is always greater than the expected return from the single optimal Kelly wager. The expected return, ER2, from the combination wager is given by

$$
E R 2=p(s a-v)+q(v b-s)-(1-p-q)(s+v)=s \mu+v \lambda
$$


Therefore

$$
E R 2=\frac{a \lambda^{2}+2 \lambda \mu+b \mu^{2}}{a b-1}
$$

The expected return, ER1, of a single Kelly wager is given by

$$
E R 1=j p a-(1-p) j=\frac{\mu^{2}}{a}
$$

The difference in expected returns between the combination wager and single wager is positive and is given by

$$
E R 2-E R 1=\frac{(a \lambda+\mu)^{2}}{a(a b-1)}>0
$$

It is this property (11) that provides an explanation of why the combination bet may be preferred to the single Kelly wager. ${ }^{5}$ From a diversification or portfolio perspective, the wager on the outcome with negative expected return has negative covariance with the wager on the outcome with positive expected return. Optimally combining two or more wagers can result in a composite wager (portfolio) with higher overall expected return and higher expected utility. ${ }^{6} 7$

\section{Conclusions}

We have shown that a utility maximizing agent with an everywhere concave utility function may optimally wager on more than one outcome in the same event even though some outcomes have negative expected rates of return to a unit stake. In future research, it would perhaps be interesting to examine how to compute the results for betting with more than three competitors such as in a typical horse race. It is also of potential interest to analyse the case of sequential rather than simultaneous realisation of the outcomes and how that compares to the simultaneous realisation case when the stake size is optimally determined. 


\section{References}

Bell, D. E. (1988). The value of pre-decision side bets for utility maximizers. Management Science, 34(6), 797-800.

Buraimo, B., Peel, D., \& Simmons, R. (2016). Positive expected returns from combination wagering: Evidence from English football. Mimeo, Lancaster University.

Johnson, J. E. V., Jones, O., \& Tang, L. (2006). Exploring decision makers use of price information in a speculative market. Management Science, 52(6), 897-908.

Phatarfod, R. (2007). Some aspects of gambling with the Kelly criterion. Mathematical Scientist, 32(1), 23-31.

Whitrow, C. (2007). Algorithms for optimal allocation of bets on many simultaneous events. Journal of the Royal Statistical Society: Series C (Applied Statistics), 56(5), 607-623.

\footnotetext{
${ }^{1}$ As an example of Bell's insight, suppose the individual has wealth of $£ 10000$ and a take it or leave it offer of a binary wager at odds of $2 / 1$. The probability of win is 0.5 and bet size is $£ 5000$. Consequently, the individual either wins $£ 10000$ or loses $£ 5000$. With log utility the individual is indifferent since $\ln (10,000)=$ $0.5 \ln (20000)+0.5 \ln (5000)$. However, a pre-decision side wager of $£ 643.6$ on evens at European Roulette, with expected return to a unit wager of $-1 / 37$, and win probability, $18 / 37$, would maximize expected utility of the composite wager since $18 / 37[0.5 \ln (20643.6)+0.5 \ln (5643.6)]+19 / 37 \ln (9356.4)>\ln (10000)$ ${ }^{2}$ As is well known, if wager size is optimally chosen, an individual with EU preferences will always derive positive expected utility from a binary wager on an outcome in an event for which the expected return to the unit wager is positive. The Kelly wager (Kelly, 1956), derived on the assumption of an EU maximizer with a logarithmic utility function, is a well-known example of the optimal stake and has been widely employed in empirical studies (Johnson et al., 2006).

${ }^{3}$ In common with other research on wagering markets we assume the decision over the amount of wealth allocated to wagering is predetermined presumably by a portfolio decision involving the expected returns and co-variances of other assets.

${ }^{4}$ Pre-decision side bets are not feasible with CARA utility in the analysis of Bell (1998). Combination wagers are feasible with a CARA utility function and given by $s=\ln [p(a b-1) /(1+b)(1-q-p)] / r(1+a)$, $v=(\ln [(q(a b-1)) /((1+a)(1-p-q))]) /(r(1+b))$.

${ }^{5}$ We can also show that the expected return per total amount staked is always lower for the combination wager than the single Kelly wager.

${ }^{6}$ It is not possible to provide a simple tractable analysis of the difference between the variance of returns of the combination and single wager. However, it is easy to create examples where the variance of the expected return from the combination wager can be greater or less than the single Kelly wager. Clearly, when variance is higher, the higher expected rate of return of the combination wager more than compensates for the higher variance of the dual bet.

${ }^{7}$ We have employed this combination wager on English Premier League soccer matches in Buraimo et al (2016).
} 